Tecno Lógicas

ISSN 0123-7799

Vol. 18, No. 34, pp. 51-62

Enero-junio de 2015

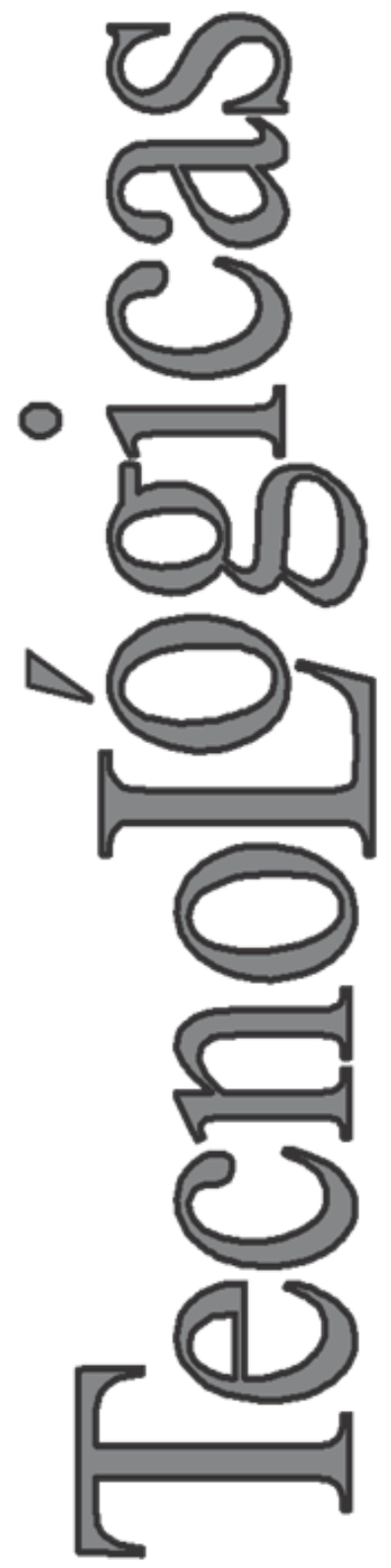

(C) Copyright 2015 por autores y Tecno Lógicas Este trabajo está licenciado bajo una Licencia Internacional Creative Commons Atribución (CC BY)

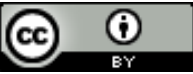

\section{Influencia de la temperatura de compactación sobre la resistencia de un material granular estabilizado con asfalto en caliente tipo MGEA_A}

\section{Influence of compaction temperature on the resistance of a stabilized granular material with asphalt cement}

\author{
Hugo A. Rondón-Quintana ${ }^{1}$, \\ Carlos F. Urazán-Bonells ${ }^{2}$ y Saieth B. Chaves-Pabón ${ }^{3}$
}

Recibido: 18 de julio de 2014, Aceptado: 15 de septiembre de 2014

Cómo citar / How to cite

H. A. Rondón-Quintana, C. F. Urazán-Bonells y S. B. Chaves-Pabón, "Influencia de la temperatura de compactación sobre la resistencia de un material granular estabilizado con asfalto en caliente tipo MGEA_A”, Tecno Lógicas, vol. 18, no. 34, pp. 51-62, 2015.

1 Doctor en Ingeniería, Facultad del Medio Ambiente y Recursos Naturales, Universidad Distrital Francisco José de Caldas, Bogotá-

Colombia, harondonq@udistrital.edu.co

2 Doctor en Gestión Territorial e Infraestructura del Transporte, Departamento de Ingeniería Civil, Facultad de Ingeniería, Universidad de La Salle, Bogotá-Colombia, caurazan@unisalle.edu.co

3 Doctor en Ciencia y Tecnología de la Ingeniería Geodésica y Cartográfica, Departamento de Ingeniería Civil, Facultad de Ingeniería, Universidad Católica de Colombia, Bogotá-Colombia, sbchaves@ucatolica.edu.co 


\section{Resumen}

En el artículo se presentan los resultados de una fase experimental cuyo principal objetivo fue evaluar la influencia de la temperatura de compactación sobre la resistencia mecánica y la susceptibilidad al agua de un material granular estabilizado con asfalto en caliente (MGEA). Lo anterior, debido a que en climas templados o de baja temperatura, se ha reportado en obra disminución de la temperatura de compactación con respecto a la óptima de laboratorio de hasta $30^{\circ} \mathrm{C}$. Adicionalmente, las especificaciones técnicas de construcción no recomiendan un rango de temperaturas de compactación para el control de este material en proyecto viales. Lo anterior genera, que en muchas ocasiones, estos materiales sean compactados de manera empírica con temperaturas que tal vez no sean las adecuadas. Las mezclas fueron fabricadas utilizando dos cementos asfálticos (CA 6070 y CA 80-100) y fueron compactadas bajo temperaturas de $90,100,110$ y $120^{\circ} \mathrm{C}$, siendo esta última la óptima de referencia. Los ensayos ejecutados fueron el Marshall, módulo resiliente, deformación permanente y tracción indirecta sobre muestras secas y sumergidas en agua. Como conclusión general se reporta, que la disminución de la temperatura de compactación de la mezcla MGEA, genera una disminución notable en su resistencia bajo carga monotónica y cíclica e incrementa el daño por humedad.

\section{Palabras clave}

Material granular ligado con asfalto en caliente, temperatura de compactación, resistencia bajo carga monotónica y cíclica, daño por humedad, MGEA.

\section{Abstract}

The influence of compaction temperature on the mechanical resistance and susceptibility to water of a bound granular material with asphalt cement (called MGEA) was evaluated in laboratory. The above, because in temperate climates or low temperature, has been reported in some road projects, lowering the temperature of compaction with respect to the laboratory optimum to $30^{\circ} \mathrm{C}$. Additionally, the technical construction specifications not recommend a range of compaction temperatures for the control of this material in road projects. This generates, that these materials are compacted empirically with temperatures that may not be appropriate. Two asphalt cements (AC 60-70 and AC 80-100) were used to prepare the MGEA mixtures. The compaction temperatures used were $105,115,125$ and $135^{\circ} \mathrm{C}$. Marshall, resilient modulus, permanent deformation and indirect traction tests were performed. The decrease of the compaction temperature produces a remarkable decrease in resistance under monotonic and cyclic loading, and increases moisture damage of the MGEA mixture tested.

\section{Keywords}

Bound granular material with asphalt cement, compaction temperature, resistance under monotonic and repeated loading, moisture damage, MGEA. 


\section{INTRODUCCIÓN}

De acuerdo con ASOPAC (Asociación de Productores y Pavimentadores Asfálticos de Colombia) [1], la mayor parte de las estructuras que se diseñan y construyen en Colombia y el mundo son flexibles o cuentan con rodaduras o superficies construidas con capas asfálticas. Según ellos, más del 90\% de las vías en el mundo están construidas en pavimento asfáltico. Para la construcción de este tipo de estructuras, en algunas ocasiones, el agregado pétreo que se explota en canteras no cumple los requisitos mínimos de calidad que exigen las especificaciones técnicas de construcción (p.e., Instituto de Desarrollo Urbano - IDU [2], Instituto Nacional de Vías - INVÍAS [3]) para conformar capas granulares de sub-base y base, generando la necesidad de estabilizar estos últimos materiales durante la construcción de obras viales, adicionándoles y ligándolos principalmente con cemento asfáltico (CA) o hidráulico. Cuando el ligante que se utiliza para cohesionar los granulares es el CA, la mezcla asfáltica resultante adquiere la denominación de material granular estabilizado con asfalto en caliente (MGEA, IDU [2], artículo 42211). Adicionalmente, el uso de estos materiales ha venido creciendo en los últimos años como reemplazo de las bases o subbases granulares no tratadas para soportar la capa asfáltica en pavimentos flexibles, principalmente en proyectos donde se necesitan plataformas más rígidas y menos permeables, o en zonas donde extender y compactar granulares se dificulte (p.e., zonas de alta precipitación).

Uno de los principales problemas que tiene la construcción de este material granular estabilizado en climas templados como la ciudad de Bogotá D.C., es que el decaimiento de la temperatura, desde su fabricación hasta su compactación, puede ser de hasta $30^{\circ} \mathrm{C}$. Lo anterior debido principalmente a: (i) El clima de la ciudad de Bogotá D.C. es predominantemente frío con temperaturas mínimas y máximas promedio de $5^{\circ} \mathrm{C}$ y $19^{\circ} \mathrm{C}$ respectivamente, humedad entre el $60 \%$ al $100 \%$, altura promedio sobre el nivel del mar de $2640 \mathrm{~m}$ y presencia de lluvias periódicas en cualquier momento del día; (ii) El contenido de CA que se utiliza para cohesionar el agregado pétreo es pequeño (generalmente entre $3,0 \%$ y $4,0 \%$ con respecto a la masa de los agregados), en comparación con otros tipos de mezcla (p.e., concreto asfáltico); (iii) Las distancias desde las plantas de producción de mezcla asfáltica hasta el sitio de la obra son grandes. Adicionalmente, la ciudad presenta problemas de congestión vehicular y movilidad. En algunas ocasiones, las volquetas transportadoras del material pueden demorar entre 1 y 2 horas desde la planta de asfaltos hasta el sitio de obra; (iv) Bogotá D.C. es una ciudad con alta nubosidad que experimenta lluvias periódicas durante el día. Bajo estas condiciones climáticas, las volquetas pueden permanecer en el sitio de la obra entre 30 minutos y 8 horas antes de que el interventor permita al contratista la extensión de la mezcla, previo al secado de la superficie humedecida por la lluvia; (v) Constructivamente, es muy común que se extienda el material en un gran tramo de vía antes de su compactación. La mezcla puede durar extendida entre 10 y 20 minutos antes de su compactación.

El problema durante el proceso constructivo de pavimentos es que las especificaciones técnicas no reportan una temperatura máxima o mínima para el control de calidad de este material. La hipótesis que soporta la ejecución de este estudio es que la disminución de la temperatura de compactación debe generar cambios en las propiedades físicas y mecánicas de la mezcla tipo MGEA. De acuerdo con Rondón et al. [4], estudios sobre el efecto de la temperatura de compactación en las propiedades físicas y mecánicas de mezclas asfálticas en caliente convencionales (sin aditivos) y modificadas han sido ampliamente reportados desde los años 50 [5]-[9]. La mayor parte de dichos estudios reporta un dete- 
rioro de la respuesta mecánica de las mezclas analizadas cuando decae la temperatura de compactación [10], [11]). Por ejemplo, Newcomb et al. [12] reportaron un incremento lineal en el módulo resiliente de mezclas asfálticas, cuando aumentó la temperatura de compactación. Aschenbrener y Far [13] reportaron un incremento en la resistencia a la deformación permanente de mezclas con el aumento en dicha temperatura. De la misma forma, Azari et al. [14] reportaron un incremento en las propiedades de resistencia al corte. Sin embargo, en este último estudio no se obtuvieron variaciones significativas en la resistencia a fatiga, evaluada bajo el modo de carga de deformación controlada. Otros estudios concluyen que el efecto de la temperatura de compactación es insignificante sobre las propiedades de las mezclas [15][17]. Crawley [18], por ejemplo, evaluó la respuesta que experimentaron mezclas asfálticas construidas in situ, compactándolas a temperaturas de $149^{\circ} \mathrm{C}$ y $107^{\circ} \mathrm{C}$. Después de 3,5 años, se extrajeron muestras de las vías pavimentadas, encontrando propiedades similares. También se reporta de manera general, que la influencia de la temperatura de compactación sobre las propiedades de mezclas asfálticas en caliente, depende del tipo de ligante asfáltico utilizado y principalmente de la granulometría empleada [9].

El objetivo del presente estudio fue evaluar el cambio que experimenta la resistencia mecánica y el daño por humedad de una mezcla asfáltica tipo MGEA, bajo diferentes temperaturas de compactación. Lo anterior, como ya se mencionó, debido a que en la ciudad de Bogotá D.C. se han reportado en obra disminución de la temperatura de las mezclas, desde su fabricación en la planta de asfaltos hasta su extensión y compactación, de hasta $30^{\circ} \mathrm{C}$. Para evaluar la resistencia de la mezcla se ejecutaron ensayos Marshall (AASHTO T 245-97, 04, INV.-E-748-13), módulo resiliente (ASTM D 4123-82, INV.E-749-13) y deformación permanente (NLT 173-84,
INV.-E-756-13), y para evaluar el daño por humedad, se ejecutaron ensayos de tracción indirecta sobre muestras secas y sumergidas en agua (ASTM D 4867/D4867M96, INV.-E-725-13). La mezcla analizada, fue fabricada empleando los dos cementos asfálticos que se producen en Colombia (CA 60-70 y CA 80-100). Adicionalmente, se empleó la granulometría de la mezcla MGEA_A y fueron compactadas bajo temperaturas de $90^{\circ} \mathrm{C}, 100^{\circ} \mathrm{C}, 110^{\circ} \mathrm{C}$ y $120^{\circ} \mathrm{C}$, siendo esta última, la temperatura de compactación inicial o de referencia de las mezclas en laboratorio. La granulometría analizada, es una de las tres que se reportan en la especificación IDU [2]. Este tipo de mezcla y granulometría fue la escogida, debido a que es la más utilizada en obra y puede ser empleada como capa de base o sub-base en estructuras flexibles para cualquier nivel de tráfico. Un estudio similar, con la granulometría especificada y empleando los dos asfaltos colombianos no se ha reportado a la fecha.

\section{METODOLOGíA}

\subsection{Caracterización de materiales}

En la Tabla 1 se presentan los valores obtenidos de los ensayos de caracterización ejecutados sobre el agregado pétreo. Se observa en la Tabla 1, que los valores de cada uno de los ensayos cumplen con el requisito mínimo de calidad exigido por la especificación IDU [2] (artículo 422-11), para fabricar mezclas tipo MGEA_A. La granulometría utilizada para la fabricación de la mezcla tipo MGEA_A, se presenta en la Tabla 2. Esta granulometría es acorde con la estipulada en la especificación IDU [2] (artículo 422-11). Para cumplir con la granulometría especificada se modificó la composición original de los agregados pétreos, tomando como referencia los valores promedios en porcentaje de la franja granulométrica que exige la especificación para la elaboración de la mezcla. 
Influencia de la temperatura de compactación sobre la resistencia de un material granular estabilizado...

\begin{tabular}{cccc} 
Tabla 1. Caracterización del agregado pétreo. Fuente: Autores & \\
\hline Ensayo & Método & Especificado & Resultado \\
\hline Peso específico (gruesos y finos) & ASTM D 854-00 & - & 2,65 \\
Equivalente de arena & ASTM D 2419-95 & $20 \%$ mín. & $65 \%$ \\
Caras fracturadas (1 cara) & ASTM D 5821-01 & $85 \%$ mín. & $91 \%$ \\
Caras fracturadas (2 caras) & ASTM D 5821-01 & $60 \%$ mín. & $82 \%$ \\
Azul de metileno & AASHTO TP 57-01 & $10 \%$ máx. & $3,4 \%$ \\
Contenido de impurezas & NLT 172/86 & - & $0,1 \%$ \\
Angularidad & ASTM C 1252-98 & $35 \%$ mín. & $59,8 \%$ \\
Índice de alargamiento & NLT 354-91 & $35 \%$ máx. & $6,0 \%$ \\
Límite líquido & ASTM D 4318-00 & $35 \%$ máx. & $9,0 \%$ \\
Índice de plasticidad & ASTM D 4318-00 & No plástico & No plástico \\
Ataque en sulfato de magnesio & ASTM C 88-99a & $18 \%$ máx. & $12,1 \%$ \\
Microdeval & ASTM D6928-03 & $20 \%$ máx. & $23,6 \%$ \\
10\% de finos, relación húmedo/seco & DNER-ME 096-98 & $75 \%$ mín. & $78 \%$ \\
RBR al 95\% de densidad seca máxima & ASTM C 131-01 & $35 \%$ máx. & $23,7 \%$
\end{tabular}

Tabla 2. Granulometría agregados para MGEA_A.

\begin{tabular}{ccc}
\multicolumn{3}{c}{ Fuente: Autores } \\
\hline Normal & Alterno & \% que pasa \\
\hline $37,5 \mathrm{~mm}$ & $11 / 2$ ” & 100 \\
$25,0 \mathrm{~mm}$ & $1 ”$ & $67-100$ \\
$19,0 \mathrm{~mm}$ & $3 / 4 ”$ & $59-91$ \\
$9,5 \mathrm{~mm}$ & $3 / 8 ”$ & $42-75$ \\
$4,75 \mathrm{~mm}$ & No. 4 & $30-60$ \\
$2,00 \mathrm{~mm}$ & No. 10 & $20-45$ \\
$0,425 \mathrm{~mm}$ & No. 40 & $10-27$ \\
$0,18 \mathrm{~mm}$ & No. 80 & $6-20$ \\
$0,075 \mathrm{~mm}$ & No. 200 & $5-15$ \\
\hline
\end{tabular}

Con base en los resultados de desgaste en la máquina de Los Ángeles, Micro-Deval y $10 \%$ de finos se reporta que el agregado pétreo grueso empleado para la fabricación de las mezclas asfálticas del presente estudio es de buena resistencia al desgaste por impacto y por abrasión, así como al fracturamiento de partículas. Así mismo, se observa que dicho agregado pétreo es de alta resistencia a desintegrarse cuando dentro de sus poros, el agua se expande por congelamiento (ensayo de sanidad en sulfato de sodio) y presenta partículas con formas ideales (redondeadas con caras angulares y fracturadas con muy poco contenido de partículas alargadas y aplanadas) para desarrollar un buen esqueleto granular que ayude en procesos de compactación de mezclas asfálticas, así como a resistir cargas estáticas, dinámicas o de impacto. Adicionalmente, se observa que las partículas finas no presentan contenido de arcilla, materia orgánica en exceso o de polvo que generen disminución de adherencia entre el agregado pétreo y el asfalto, y disminución de resistencia y durabilidad de la mezcla por exceso de finos o contaminantes.

A los cementos asfálticos CA 60-70 y CA 80-100, se les realizaron los ensayos que exigen las especificaciones IDU [2] e INVIAS [3] para caracterizarlos, y los resultados se presentan en la Tabla 3 . Se observa que los asfaltos cumplen con los requisitos mínimos de calidad que exigen las especificaciones técnicas para ser empleados como ligantes en la fabricación de mezclas MGEA_A. En las Tablas 4 y 5 se presenta la caracterización reológica de los asfaltos a temperaturas altas e intermedias de servicio usando un reómetro dinámico de corte - DSR (AASHTO T 315-05). El grado de desempeño a altas e intermedias temperaturas de servicio es $58^{\circ} \mathrm{C}$ $\left(\left|\mathrm{G}^{*}\right| / \sin \delta>1.0 \mathrm{kPa}\right.$ para asfalto sin envejecer y $\left(\left|\mathrm{G}^{*}\right| / \sin \delta>2,2 \mathrm{kPa}\right.$ para asfalto envejecido en RTFOT) y $22^{\circ} \mathrm{C}\left(\left|\mathrm{G}^{*}\right| \sin \delta<\right.$ 
Influencia de la temperatura de compactación sobre la resistencia de un material granular estabilizado...

$5000 \mathrm{kPa}$ para asfalto envejecido en $\mathrm{RTFOT}+\mathrm{PAV}$ ) respectivamente. $\mathrm{G}^{*}$ es el módulo complejo de corte y $\delta$ el ángulo de fase. El asfalto CA 60-70 presenta propie- dades físicas y reológicas que lo hacen ser más rígido y viscoso en comparación con el CA 80-100.

Tabla 3. Características generales de los asfaltos. Fuente: Autores

\begin{tabular}{ccccc}
\hline Ensayo & Método & Unidad & CA 60-70 & CA 80-100 \\
\hline & Ensayos sobre el asfalto sin envejecer & & \\
\hline Penetración $\left(25^{\circ} \mathrm{C}, 100 \mathrm{~g}, 5 \mathrm{~s}\right)$ & ASTM D-5 & $0,1 \mathrm{~mm}$ & 65 & 83 \\
Índice de penetración & NLT 181/88 & - & $-0,8$ & $-0,6$ \\
Viscosidad absoluta $\left(60^{\circ} \mathrm{C}\right)$ & ASTM D-4402 & Poises & 1800 & 1450 \\
Gravedad específica & AASHTO T 228-04 & - & 1,016 & 1,011 \\
Ductilidad $\left(25^{\circ} \mathrm{C}, 5 \mathrm{~cm} / \mathrm{min}\right)$ & ASTM D-113 & $\mathrm{cm}$ & $>105$ & $>105$ \\
Punto de ablandamiento & ASTM D-36-95 & 50 & 48 \\
Solubilidad en Tricloroetileno & ASTM D-2042 & $\%$ & $>99$ & $>99$ \\
Contenido de agua & ASTM D-95 & $\% 0,2$ & $<0,2$ \\
Punto de inflamación & ASTM D-92 & 285 & 300 \\
\hline Pérdida de masa & Ensayos después del residuo en RTFOT & & \\
\hline Penetración $\left(25^{\circ} \mathrm{C}, 100 \mathrm{~g}, 5 \mathrm{~s}\right)$ & ASTM D-2872 & $\%$ & 0,6 & 0,7 \\
\hline
\end{tabular}

Tabla 4. Caracterización reológica de CA 60-70. Fuente: Autores

\begin{tabular}{|c|c|c|c|c|c|c|}
\hline Temperatura $\left[{ }^{\circ} \mathrm{C}\right]$ & Frecuencia $[\mathrm{rad} / \mathrm{s}]$ & $\delta\left[^{\circ}\right]$ & $G^{*}[\mathrm{~Pa}]$ & $\left|G^{*}\right| / \sin \delta[\mathrm{kPa}]$ & $\left|G^{*}\right| \sin \delta[\mathrm{kPa}]$ & Viscosidad $[\mathrm{Pa} \cdot \mathrm{s}]$ \\
\hline \multicolumn{7}{|c|}{ CA 60-70 sin envejecer } \\
\hline 58 & 10 & 87 & 2470 & 2,473 & 2,467 & 245 \\
\hline 64 & 10 & 88 & 1002 & 1,000 & 1,000 & 105 \\
\hline 70 & 10 & 89 & 453 & 0,453 & 0,453 & 49 \\
\hline \multicolumn{7}{|c|}{ CA 60-70 envejecido en RTFOT } \\
\hline 52 & 10 & 83 & 11062 & 11,15 & 10,98 & 1022 \\
\hline 58 & 10 & 85 & 4276 & 4,29 & 4,26 & 416 \\
\hline 64 & 10 & 87 & 1701 & 1,70 & 1,70 & 138 \\
\hline \multicolumn{7}{|c|}{ CA 60-70 envejecido en RTFOT + PAV } \\
\hline 16 & 10 & 44 & 14266000 & 20536,7 & 9910,0 & 967610 \\
\hline 19 & 10 & 45 & 10193000 & 14415,1 & 7207,6 & 720080 \\
\hline 22 & 10 & 47 & 6659000 & 9105,0 & 4870,0 & 488890 \\
\hline
\end{tabular}

Tabla 5. Caracterización reológica de CA 80-100. Fuente: Autores

\begin{tabular}{|c|c|c|c|c|c|c|}
\hline Temperatura $\left[{ }^{\circ} \mathrm{C}\right]$ & Frecuencia $[\mathrm{rad} / \mathrm{s}]$ & $\delta\left[^{\circ}\right]$ & $G^{*}[\mathrm{~Pa}]$ & $\left|G^{*}\right| / \sin \delta[\mathrm{kPa}]$ & $\left|G^{*}\right| \sin \delta[\mathrm{kPa}]$ & Viscosidad $[\mathrm{Pa} \cdot \mathrm{s}]$ \\
\hline \multicolumn{7}{|c|}{ CA 80-100 sin envejecer } \\
\hline 58 & 10 & 87 & 1980 & 1,98 & 1,98 & 212 \\
\hline 64 & 10 & 88 & 776 & 0,78 & 0,78 & 89 \\
\hline 70 & 10 & 89 & 413 & 0,41 & 0,41 & 39 \\
\hline \multicolumn{7}{|c|}{ CA 80-100 envejecido en RTFOT } \\
\hline 52 & 10 & 84 & 9150 & 9,20 & 9,10 & 913 \\
\hline 58 & 10 & 85 & 2698 & 2,71 & 2,69 & 387 \\
\hline 64 & 10 & 88 & 1456 & 1,46 & 1,46 & 109 \\
\hline \multicolumn{7}{|c|}{ CA 80-100 envejecido en RTFOT + PAV } \\
\hline 16 & 10 & 47 & 11672000 & 15959,4 & 8536,4 & 858820 \\
\hline 19 & 10 & 49 & 8534000 & 11307,6 & 6440,7 & 671490 \\
\hline 22 & 10 & 52 & 5802000 & 7362,8 & 4572,1 & 406330 \\
\hline
\end{tabular}


Influencia de la temperatura de compactación sobre la resistencia de un material granular estabilizado...

\subsection{Diseño de mezcla asfáltica MGEA_A}

De acuerdo con IDU [2], el diseño de materiales granulares estabilizados con asfalto en caliente (MGEA) se realiza por medio del método Marshall (INV.-E-74813, AASHTO T 245-97, 04), y la obtención del porcentaje óptimo de cemento asfáltico se obtiene al alcanzar una estabilidad $(E)$ mínima de $750 \mathrm{~kg}$ y un flujo $(F)$ comprendido entre $2 \mathrm{~mm}$ y $4 \mathrm{~mm}$ cuando las briquetas se compactan a 75 golpes por cara. Consideraciones adicionales para el diseño de este tipo de mezcla son: (i) La resistencia a tracción de la mezcla [19] (INV.-E725-13, ASTM D 4867/D4867M-96) en curado húmedo debe ser al menos el 70\% de la alcanzada bajo condición seca; (ii) $\mathrm{El}$ módulo resiliente [19] (INV.-E-749, ASTM D 4123-82) debe ser superior a $2000 \mathrm{MPa}$ para una temperatura ambiente de $20^{\circ} \mathrm{C}$ y una frecuencia de carga de $10 \mathrm{~Hz}$.

Luego de realizar los ensayos al agregado pétreo y a los cementos asfálticos, se fabricaron cinco briquetas (compactadas a 75 golpes por cara) para cada porcentaje de asfalto de $3,0 \%, 3,5 \%, 4,0 \%$ y $4,5 \%$, con el fin de realizar el diseño Marshall. La temperatura de mezcla y compactación de las briquetas fue de $125^{\circ} \mathrm{C}$ y $120^{\circ} \mathrm{C}$ respectivamente. Estas temperaturas fueron escogidas con base en el ensayo de viscosidad realizado a los cementos asfálticos, cumpliendo con el rango especificado para mezclas del tipo abierto (viscosidad de mezcla recomendada entre $700 \mathrm{cp}$ y $900 \mathrm{cp})$. En las Tablas 6 y 7 se presenta el resumen del ensayo Marshall para las mezclas fabricadas con CA 60-70 y CA 80-100 respectivamente.

Sobre briquetas fabricadas con los mismos porcentajes de asfalto y temperaturas de mezcla y compactación, se realizó adicionalmente el ensayo de resistencia a tracción de la mezcla [19] (ASTM D 4867/D4867M-96, INV.-E-725-13) en curado seco y húmedo. La relación de esfuerzos de tracción húmedo/seco (TSR, en \%) se reporta en la Tabla 8.
Tabla 6. Resumen del ensayo Marshall para mezcla asfáltica MGEA_A fabricada con CA 60-70. Fuente: Autores

\begin{tabular}{cccccc}
\hline CA & $\begin{array}{c}\text { Densidad } \\
{[\%]}\end{array}$ & $\begin{array}{c}E \\
{[\mathrm{~g} / \mathrm{cm} 3]}\end{array}$ & $\begin{array}{c}\text { Vacíos } \\
\text { mezcla } \\
{[\%]}\end{array}$ & $\begin{array}{c}F \\
{[\mathrm{~mm}]}\end{array}$ & $\begin{array}{c}E / F \\
{[\mathrm{~kg} / \mathrm{mm}]}\end{array}$ \\
\hline 3,0 & 2,246 & 836,7 & 11,13 & 3,15 & 265,9 \\
3,5 & 2,271 & 1034,6 & 9,46 & 3,71 & 279,2 \\
4,0 & 2,300 & 1192,5 & 7,59 & 4,42 & 270,2 \\
4,5 & 2,294 & 1062,2 & 7,11 & 4,78 & 222,6 \\
\hline
\end{tabular}

Tabla 7. Resumen del ensayo Marshall para mezcla asfáltica MGEA_A fabricada con CA 80-100. Fuente: Autores

\begin{tabular}{cccccc}
\hline $\begin{array}{c}\text { CA } \\
{[\%]}\end{array}$ & $\begin{array}{c}\text { Densidad } \\
{\left[\mathrm{g} / \mathrm{cm}^{3}\right]}\end{array}$ & $\begin{array}{c}E \\
{[\mathrm{~kg}]}\end{array}$ & $\begin{array}{c}\text { Vacíos } \\
\text { mezcla } \\
{[\%]}\end{array}$ & $\begin{array}{c}F \\
{[\mathrm{~mm}]}\end{array}$ & $\begin{array}{c}E / F \\
{[\mathrm{~kg} / \mathrm{mm}]}\end{array}$ \\
\hline 3,0 & 2,236 & 773,6 & 11,53 & 3,33 & 232,6 \\
3,5 & 2,256 & 923,7 & 10,04 & 3,61 & 256,3 \\
4,0 & 2,280 & 1043,5 & 8,39 & 4,11 & 253,8 \\
4,5 & 2,311 & 963,7 & 6,44 & 4,90 & 197,1 \\
\hline
\end{tabular}

Tabla 8. Evolución del daño por humedad para mezcla asfáltica MGEA_A. Fuente: Autores

\begin{tabular}{cccc}
\hline $\begin{array}{c}\text { CA 60-70 } \\
{[\%]}\end{array}$ & $\begin{array}{c}\text { TSR } \\
{[\%]}\end{array}$ & $\begin{array}{c}\text { CA 80-100 } \\
{[\%]}\end{array}$ & $\begin{array}{c}\text { TSR } \\
{[\%]}\end{array}$ \\
\hline 3,0 & 66,5 & 3,0 & 65,2 \\
3,5 & 75,4 & 3,5 & 71,5 \\
4,0 & 76,2 & 4,0 & 73,3 \\
4,5 & 80,3 & 4,5 & 79,6 \\
\hline
\end{tabular}

El porcentaje óptimo de cemento asfáltico, para la mezcla MGEA_A fabricada con ambos tipos de asfalto, fue de $3,5 \%$ de acuerdo con los resultados presentados en las Tablas 6 a 8. En este porcentaje se obtiene la mayor resistencia bajo carga monotónica $(E / F)$ y se cumple con los requisitos mínimos exigidos por la especificación IDU [2], para diseñar mezclas tipo MGEA.

\subsection{Desarrollo experimental para el logro de los objetivos}

Una vez realizado el diseño de la mezcla MGEA_A, se procedió a fabricar briquetas Marshall (AASHTO T 245-97, 04, INV.E-748-13) con el porcentaje óptimo de asfalto $(3,5 \%)$. La temperatura de mezcla del agregado pétreo con los asfaltos fue de $125^{\circ} \mathrm{C}$, y la de compactación, inició con $120^{\circ} \mathrm{C}$ pero luego fue disminuyendo $10^{\circ} \mathrm{C}$ 
con respecto a la del diseño, hasta alcanzar un máximo de disminución de $30^{\circ} \mathrm{C}$. Lo anterior con el fin de evaluar la respuesta que experimentan bajo carga monotónica cuando la temperatura de compactación disminuye en $30^{\circ} \mathrm{C}$. Para evaluar dicha respuesta, los parámetros principales utilizados en el análisis fueron la estabilidad (E) y la relación entre la estabilidad y el flujo $(E / F)$ obtenido del ensayo Marshall (AASHTO T 245-97, INV.-E-748-13). De la misma forma, y con el fin de evaluar la resistencia al daño por humedad, se midió la relación de esfuerzos de tracción húmedo/seco (TSR, en \%) en el ensayo de tracción indirecta (ASTM D 4867/D4867M-96, INV.-E-725-13). Para evaluar el comportamiento bajo carga cíclica fueron ejecutados ensayos de módulo resiliente (ASTM D 4123-82, INV.-E-749-13) a tres temperaturas $\left(10^{\circ} \mathrm{C}, 20^{\circ} \mathrm{C}\right.$ y $\left.30^{\circ} \mathrm{C}\right)$ y frecuencias de carga $(2,5 \mathrm{~Hz}, 5 \mathrm{~Hz}$ y $10 \mathrm{~Hz})$, y deformación permanente (NLT 173-84, INV.-E-756-13) ejecutado a $60^{\circ} \mathrm{C}$ y presión de contacto de $900 \mathrm{kPa}$. Estos últimos dos ensayos fueron ejecutados sobre muestras fabricadas con el contenido óptimo de asfalto CA 60-70 y CA 80-100, y temperaturas de compactación de $90^{\circ} \mathrm{C}$ y $120^{\circ} \mathrm{C}$.

\section{RESULTADOS Y ANÁLISIS}

\subsection{Caracterización bajo carga monotónica y daño por humedad}

En las Fig. 1 y 2 se presenta la evolución de la estabilidad (E) y la relación estabilidad - flujo $(E / F)$, con la temperatura de compactación de la mezcla estabilizada con asfalto en caliente tipo MGEA_A. Se observa una disminución aproximada de $24 \%$ en la estabilidad, cuando disminuye la temperatura de compactación desde $120^{\circ} \mathrm{C}$ hasta $90^{\circ} \mathrm{C}$ para el caso de la mezcla fabricada con CA 60-70 y CA 80-100.

De la misma forma, para las mismas condiciones, el material experimenta una disminución aproximada en la resistencia bajo carga monotónica de la mezcla $(E / F)$ de $40 \%$ y $35 \%$ cuando se fabrica con CA 80 100 y CA 60-70 respectivamente.

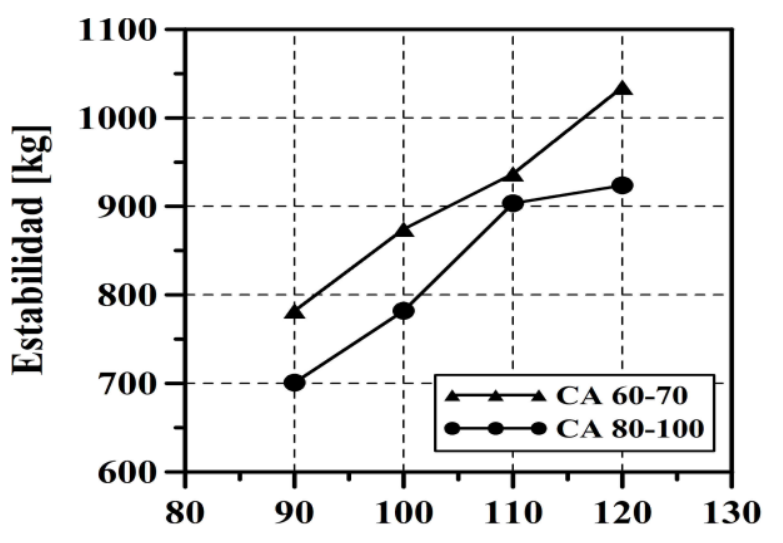

Temperatura de compactación $\left[{ }^{\circ} \mathrm{C}\right]$

Fig. 1. Evolución de $E$ con la temperatura de compactación. Fuente: Autores

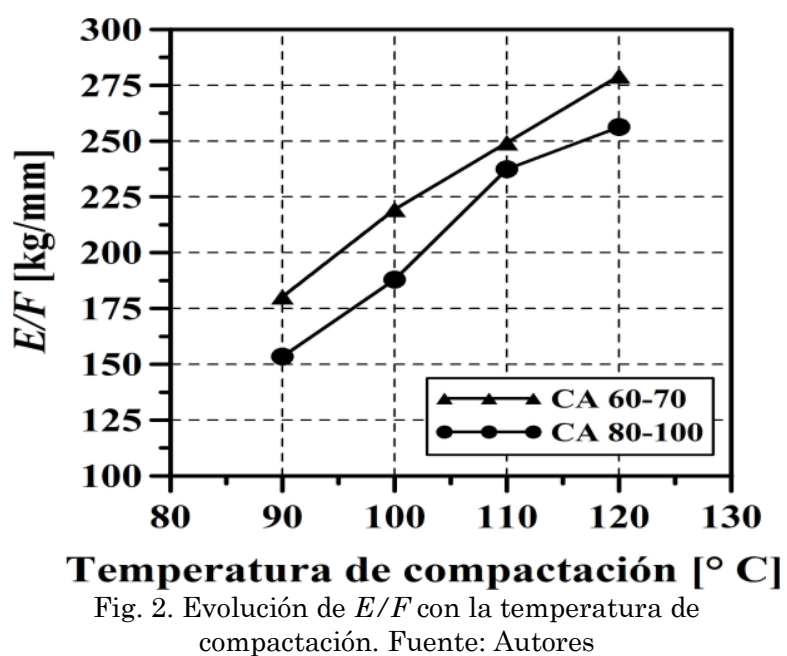

Esta disminución en la resistencia puede ser explicada en parte por el aumento aproximado de $4 \%$ que experimentó la mezcla en los vacíos con aire cuando disminuyó la temperatura de compactación (ver Fig. 3).

Este aumento en los vacíos con aire también ayudó a que el daño por humedad incrementara sobre las muestras ensayadas bajo tracción indirecta (ver Fig. 4). En la Fig. 4 se observa que las mezclas compactadas a temperaturas inferiores a $110^{\circ}$ $\mathrm{C}$ no cumplen con el criterio de resistencia al daño por humedad requerido según especificaciones de diseño. 


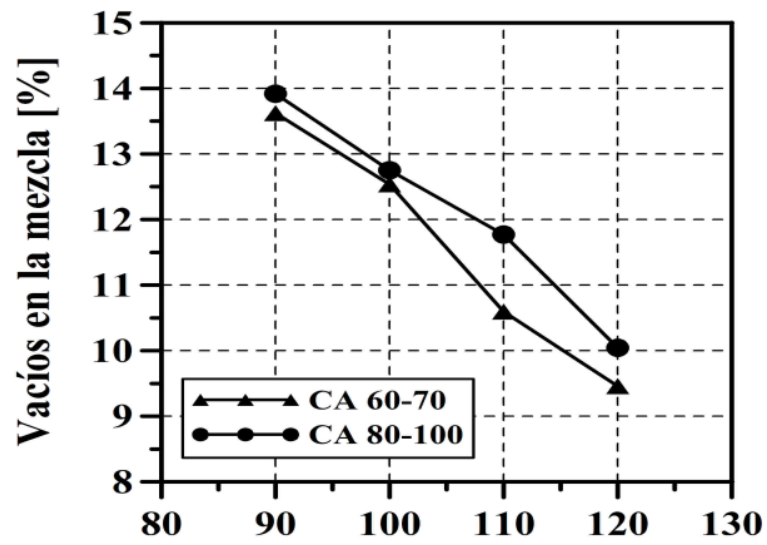

Temperatura de compactación $\left[{ }^{\circ} \mathrm{C}\right]$ Fig. 3. Evolución de los vacíos con aire con la temperatura de compactación. Fuente: Autores

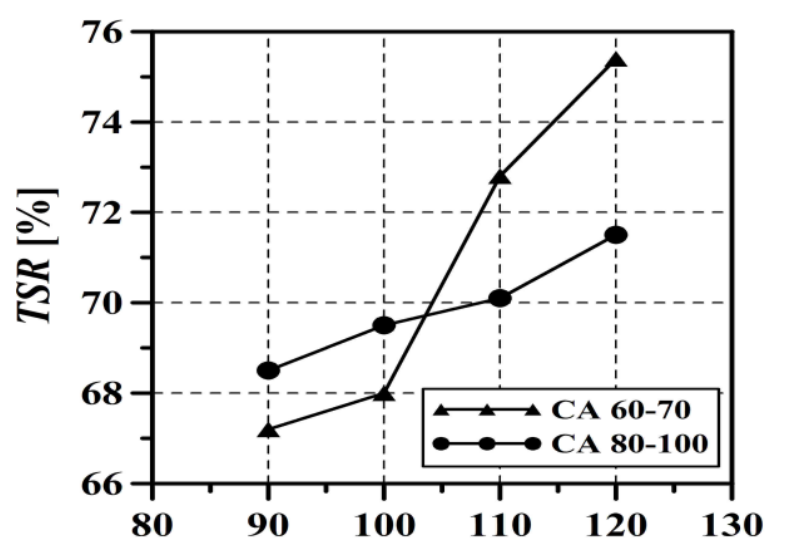

Temperatura de compactación $\left[{ }^{\circ} \mathrm{C}\right]$

Fig. 4. Evolución de la relación de esfuerzos de tracción húmedo/seco (TSR, en \%) con la temperatura de compactación. Fuente: Autores

Este aumento en los vacíos con aire es producto de la pérdida de manejabilidad y trabajabilidad de las mezclas en laboratorio al disminuir la temperatura de compactación.

\subsection{Caracterización bajo carga cíclica}

Las Fig. 5 a 7 presentan la evolución del módulo resiliente para temperatura de ensayo de $10^{\circ} \mathrm{C}, 20^{\circ} \mathrm{C}$ y $30^{\circ} \mathrm{C}$ respectivamente. Se observa, que la mezcla MGEA_A fabricada con asfalto CA 60-70 es más rígida que aquella fabricada con $\mathrm{CA} 80$ 100. Estos resultados son coherentes con los presentados en la Fig. 2, en donde la mezcla más resistente bajo carga monotónica es aquella fabricada con CA 60-70.
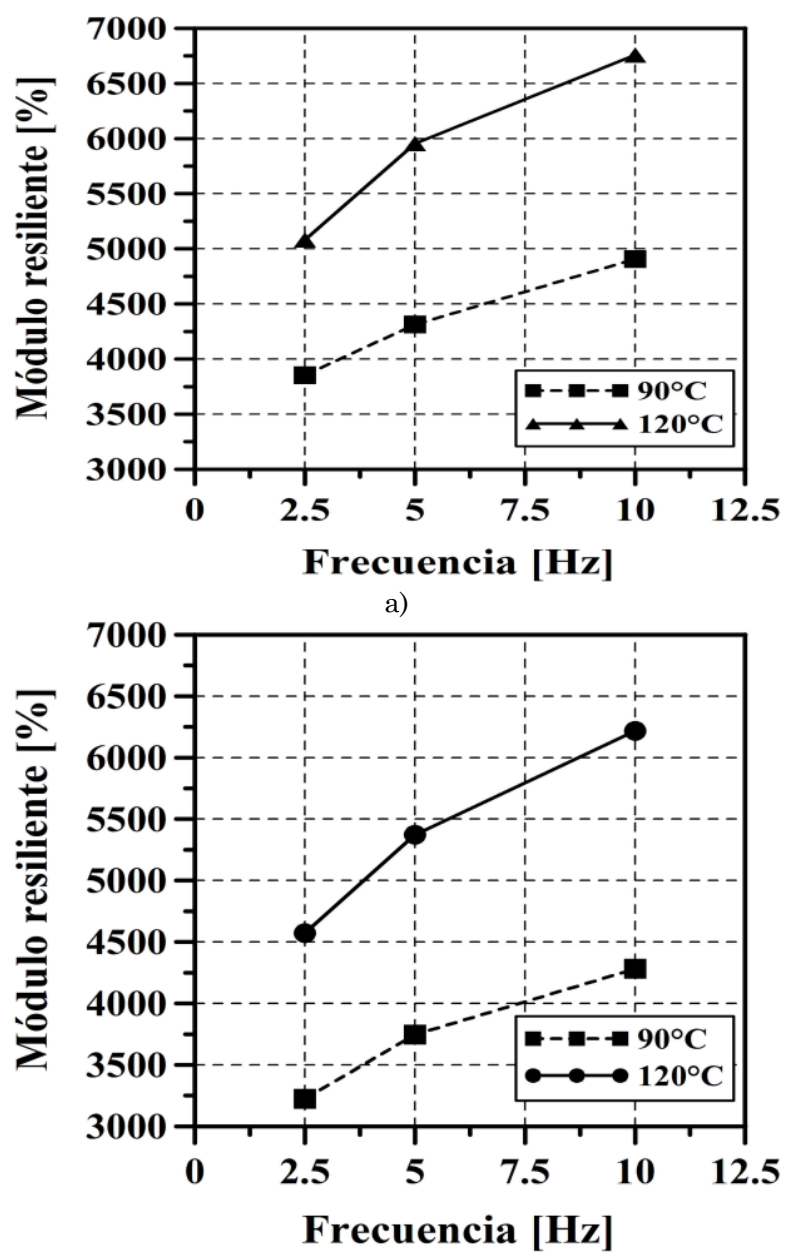

b)

Fig. 5. Evolución del módulo resiliente para una temperatura de ensayo de $10^{\circ} \mathrm{C}$, a) CA $60-70$, b) CA 80-100. Fuente: Autores

Lo anterior es debido principalmente a que el asfalto CA 60-70 es más rígido que el CA 80-100. Adicionalmente, se observa una disminución notable en la rigidez bajo carga cíclica cuando disminuye en $30^{\circ} \mathrm{C}$ la temperatura de compactación de la mezcla MGEA_A. Para el caso de la mezcla fabricada con CA 60-70, la disminución promedio de la rigidez es de $27 \%, 41 \%$ y $17 \%$ cuando la temperatura de ensayo es de $10^{\circ} \mathrm{C}, 20^{\circ} \mathrm{C}$ y $30^{\circ} \mathrm{C}$ respectivamente. Esta disminución promedio de la rigidez se encuentra entre $31 \%$ y $38 \%$ para el caso de la mezcla fabricada con CA 80-100. La Tabla 
9 presenta los resultados del ensayo de deformación permanente.
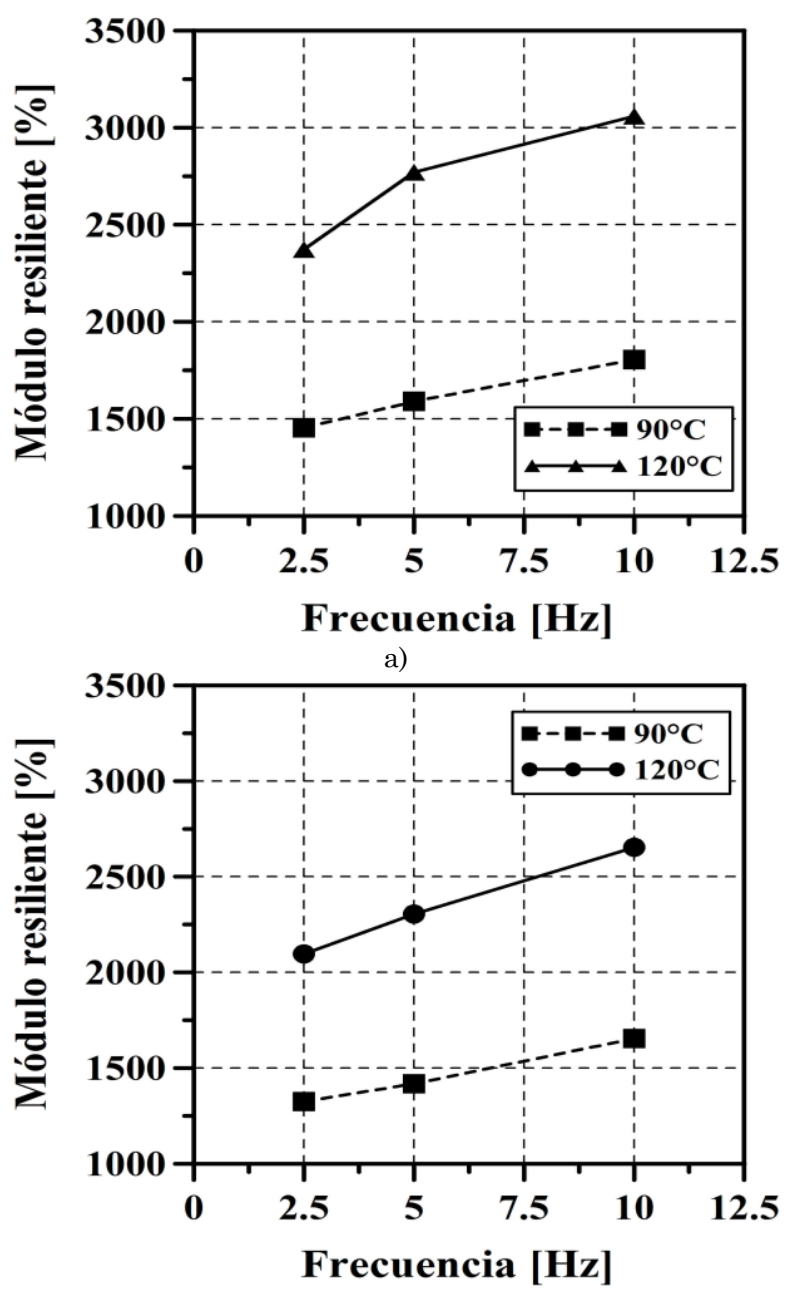

b)

Fig. 6. Evolución del módulo resiliente para una temperatura de ensayo de $20^{\circ} \mathrm{C}$, a) CA 60-70, b) CA 80-100. Fuente: Autores

Tabla 9. Resistencia a la deformación permanente. Fuente: Autores

\begin{tabular}{lcccc}
\hline \multirow{2}{*}{$\begin{array}{l}\text { Ensayo de deforma- } \\
\text { ción permanente }\end{array}$} & \multicolumn{2}{c}{$\mathrm{CA} 60-70$} & \multicolumn{2}{c}{$\mathrm{CA} 80-100$} \\
\cline { 2 - 5 } & $90^{\circ} \mathrm{C}$ & $120^{\circ} \mathrm{C}$ & $90^{\circ} \mathrm{C}$ & $120^{\circ} \mathrm{C}$ \\
\hline $\begin{array}{l}\text { Velocidad en el } \\
\text { intervalo de } 105 \mathrm{a}\end{array}$ & 13,3 & 8,1 & 14,0 & 10,2 \\
$\begin{array}{l}120 \text { minutos } \\
(\mu \mathrm{m} / \mathrm{m})\end{array}$ & & & & \\
\hline
\end{tabular}

Se observa que la mezcla más resistente al fenómeno de deformación permanente o ahuellamiento es aquella fabricada con CA 60-70. Lo anterior es coherente con los resultados reportados de rigidez bajo carga monotónica y cíclica presentados en las Fig. 2 y Fig. 5 a 7.

Adicionalmente se observa, que al disminuir la temperatura de compactación en $30^{\circ} \mathrm{C}$, la mezcla MGEA_A fabricada con ambos tipos de asfalto, incrementa la velocidad de deformación, lo que se traduce en disminución de la resistencia al ahuellamiento. Esta menor resistencia a la deformación es debida principalmente a que las mezclas aumentan sus vacíos con aire cuando disminuye la temperatura de compactación (ver Fig. 3).
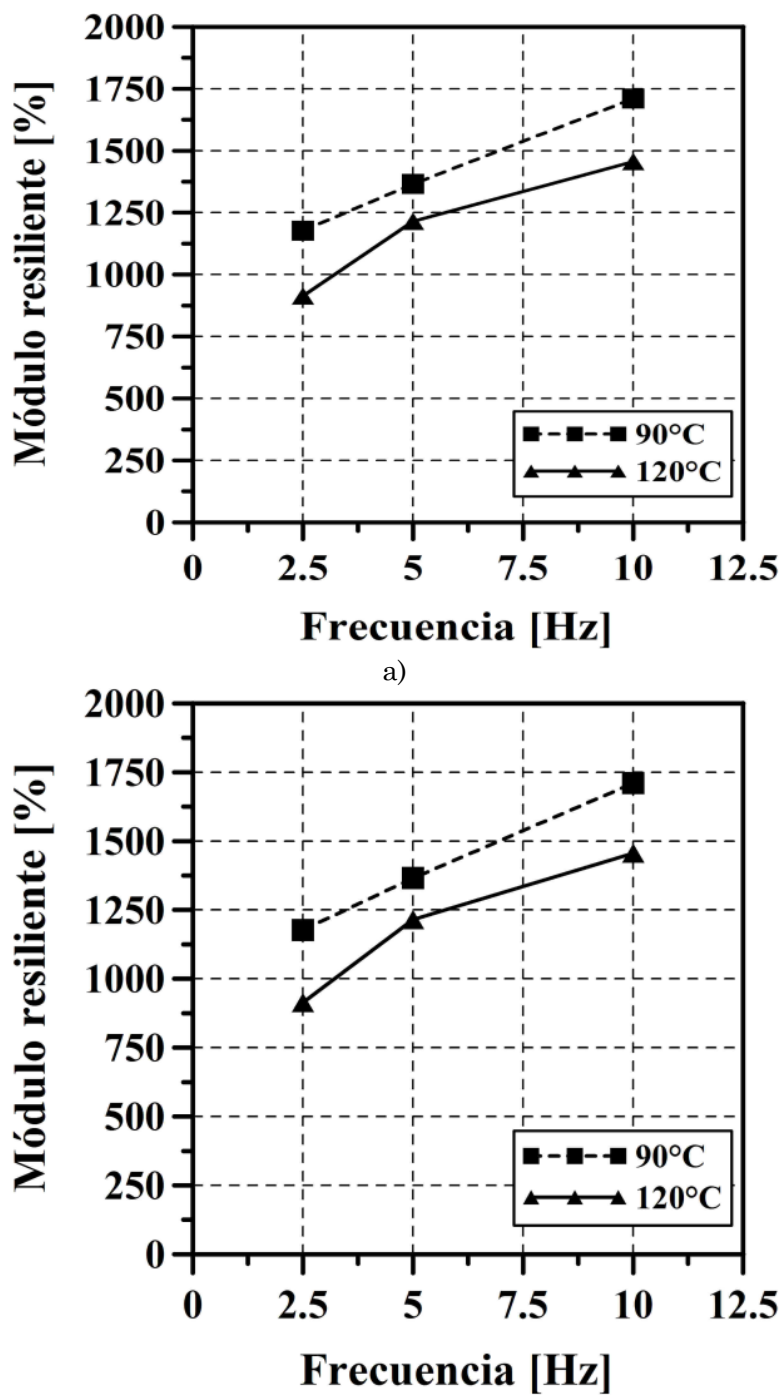

b)

Fig. 7. Evolución del módulo resiliente para una temperatura de ensayo de $30^{\circ} \mathrm{C}$, a) CA 60-70, b) CA 80-100. Fuente: Autores 


\section{CONCLUSIONES}

El presente estudió evaluó en laboratorio la influencia que tiene la disminución de la temperatura de compactación sobre el daño por humedad y la resistencia mecánica bajo carga monotónica y cíclica, de una mezcla estabilizada con asfalto en caliente tipo MGEA_A [2]. En términos generales, la mezcla tipo MGEA_A experimenta un notable decaimiento en su resistencia bajo carga monotónica, cuando disminuye la temperatura de compactación en $30^{\circ} \mathrm{C}$ con respecto a la de referencia en el laboratorio. La disminución en dicha resistencia fue de $35 \%$ y $40 \%$ cuando se fabrica con CA 60-70 y CA 80-100 respectivamente. Así mismo, el daño por humedad incrementa en la mezcla analizada. Con base en los resultados obtenidos de la relación de esfuerzos de tracción húmedo/seco - TSR se reporta, que la mezcla MGEA_A fabricada con CA 60-70 y CA 80-100 no cumple el criterio establecido de resistencia al daño por humedad cuando disminuye la temperatura de compactación $16^{\circ} \mathrm{C}$ y $11^{\circ} \mathrm{C}$ respectivamente con respecto a la de referencia de laboratorio. Lo anterior es debido principalmente al incremento en los vacíos con aire en la mezcla, dificultad de compactación de la misma al disminuir la temperatura y a la pérdida de adherencia entre el agregado pétreo y el cemento asfáltico.

Baja carga cíclica, la mezcla MGEA_A experimentó una disminución notable en su rigidez y resistencia a la deformación permanente cuando disminuyó la temperatura de compactación. Para el caso de la mezcla fabricada con CA 60-70, la disminución promedio de la rigidez fue de $27 \%$, $41 \%$ y $17 \%$ cuando la temperatura de ensayo fue de $10^{\circ} \mathrm{C}, 20^{\circ} \mathrm{C}$ y $30^{\circ} \mathrm{C}$ respectivamente, y para el caso de la mezcla fabricada con CA 80-100, esta disminución promedio se encontró entre $31 \%$ y $38 \%$. Lo anterior hace prever la necesidad de ejecutar un estricto control en laboratorio y en obra de la temperatura de compactación de mezclas MGEA.
Con base en los resultados obtenidos se concluye, adicionalmente, que la mejor respuesta como material para conformar una capa de base en una estructura de pavimento, lo experimentó la mezcla MGEA_A fabricada con CA 60-70. Lo anterior debido a que esta mezcla desarrolló mayor resistencia al daño por humedad y rigidez bajo carga monotónica y cíclica que aquella fabricada con CA 80-100. Adicionalmente, la mezcla MGEA_A fabricada con CA 60-70 es más resistente a cambiar sus propiedades cuando disminuye la temperatura de compactación. Las conclusiones reportadas en el presente artículo son derivadas de estudios ejecutados en el laboratorio, por lo anterior se hace necesaria una futura investigación que evalúe y lo correlacione con mediciones obtenidas en campo. Así mismo, se prevé una futura fase experimental para evaluar en laboratorio, la resistencia a fatiga de las mezclas analizadas.

\section{REFERENCIAS}

[1] ASOPAC, "Cartilla del Pavimento Asfáltico," Bogotá D.C., 2004.

[2] IDU, "Especificaciones Técnicas Generales de Materiales y Construcción para Proyectos de Infraestructura Vial y de Espacio Público en Bogotá D.C.," Bogotá D.C., Colombia, 2011.

[3] INVÍAS, "Especificaciones Generales para Construcción de Carreteras," Bogotá D.C., Colombia, 2013.

[4] A. M. Rondón, H. A.; Molano, Y.; Tenjo, "Influencia de la temperatura de compactación sobre la resistencia bajo carga monotónica de mezclas asfálticas modificadas con grano de caucho reciclado de llantas," Tecno Lógicas, no. 29, pp. 11-29, 2012.

[5] C. F. Parker, "Use of steel-tired rollers," Washington D.C., 1950.

[6] P. J. Serafin, L. L. Kole, and A. P. Chritz, "Michigan bituminous experimental road. Final Report," Michigan Dept. of State Highways, Michigan, 1967.

[7] R. B. Kennedy, T. W.; Roberts, F. L.; McGennis, "Effects of compaction temperature and effort on the engineering properties of asphalt concrete mixtures," ASTM Spec. Tech. Publ., no. 829, pp. 48-66, 1984. 
[8] M. J. Shiva Kumar, "Effect of varying mixing and compaction temperature on Marshall properties of bituminous concrete mix," Karnataka State, India, 2010.

[9] J. R. West, R. C.; Watson, D. E.; Turner, P. A.; Casola, "Mixing and compaction temperatures of asphalt binders in hot-mix asphalt - NCHRP Report 648," Washington D.C., 2010.

[10] F. A. Reyes, O. J.; Camacho, J. F.; Reyes, "Influencia de la temperatura y nivel de energía de compactación en las propiedades dinámicas de una mezcla asfáltica," Rev. Fac. Ing. Univ. Antioquia, no. 36, pp. 121-130, 2006.

[11] P. Covarrubias, P. L.; Ibáñez, I. C.; Miró, R.; Garnica, "Efecto de la temperatura de compactación sobre el módulo resiliente de las mezclas asfálticas," Rev. Carreteras, no. 163, pp. 8-20, 2009.

[12] J. A. Newcomb, D. E.; Stroup-Gardiner, M.; Epps, "Laboratory and field studies of polyolefin and latex modifiers for asphalt mixtures," in Polymer modified asphalt binders, ASTM STP 1108, 1992, pp. 129-150.

[13] N. Aschenbrener, T.; Far, "Influence of temperature and antistripping treatment on the results from the Hamburg Wheel-Tracking Device - Final Report," Colorado, 1994.

[14] H. Azari, R. H. McCuen, and K. D. Stuart, "Optimum Compaction Temperature for Modified Binders," J. Transp. Eng., vol. 129, no. 5, pp. 531-537, Sep. 2003.

[15] P. McGennis, R. B.; Anderson, R. M.; Perdomo, D.;Turner, "Issues pertaining to use of the Superpave Gyratory Compactor," Transp. Res. Rec., no. 1543, pp. 139-144, 1996.

[16] D. I. Bahia, H. U.; Hanson, "NCHRP Project 910 Superpave Protocols for modified asphalt binders, Draft Topical Report (Task 9)," Washington D.C., 2000.

[17] E. R. Huner, M. H.; Brown, "Effects of re-heating and compaction temperature on hot mix asphalt volumetrics, NCAT Report 01-04," Auburn, 2001.

[18]A. B. Crawley, "An evaluation of lower mixing temperatures for bituminous paving mixes, MSHD-RD-85-069," Mississippi, 1985.

[19] INVÍAS, "Normas de Ensayos de Materiales para Carreteras," Instituto Nacional de Vías, Bogotá D.C., Colombia, 2013. 